\title{
Curative effect of $\beta$-blocker on various ejection fractions of patients with atrial fibrillation
}

\author{
WEI CAO and LIKUN MA \\ Department of Cardiology, The First Affiliated Hospital of USTC, Division of Life Science and \\ Medicine, University of Science and Technology of China, Hefei, Anhui 230001, P.R. China
}

Received May 10, 2019; Accepted June 20, 2019

DOI: $10.3892 /$ etm.2019.7703

\begin{abstract}
The aim of this study was to retrospectively determine the effects of metoprolol on patients presenting with persistent atrial fibrillation (AF), but either with or without a reduced ejection fraction $(\mathrm{EF})$. All patients meeting the inclusion criteria were treated for 2 years with metoprolol. Blood pressure, heart rate and echocardiography parameters were measured and analysed in patients before and after treatment. The patients were divided into 2 cohorts as follows: Those presenting with a low EF $(<50 \%)$ and those with normal EF values $(\geq 50 \%)$. In total, 151 patients enrolled were 136 ; however, of these 15 were lost to mortality during the follow-up period, thus leaving a total of 136 patients. In total, 42 patients presented $\mathrm{EF}$ values $<50 \%$, while the remaining 94 presented with normal EF values. Treatment with metoprolol controlled blood pressure (both diastolic and systolic) and heart rate in patients with both low and normal EF values. EF values in the low EF group significantly increased following treatment. In addition, the echocardiography data revealed a statistically significant decrease in left atrial and ventricular diameters in the low EF group. On the whole, the findings of this study demonstrate that patients with $\mathrm{AF}$ and low EF values who were treated with metoprolol presented with improved cardiac function parameters. However, metoprolol should be contraindicated for patients with high EF values (i.e., absence of heart failure) as it seemed to increase their risk of heart failure based on the $\mathrm{N}$-terminal pro b-type natriuretic peptide (NT-pro BNP) results.
\end{abstract}

\section{Introduction}

Atrial fibrillation (AF) is the most common arrhythmia diagnosed in adults (1), with an increasing prevalence with advancing age (2). Epidemiological studies have reported an

Correspondence to: Dr Likun Ma, Department of Cardiology, The First Affiliated Hospital of USTC, Division of Life Science and Medicine, University of Science and Technology of China, 27 Lu Jiang Road, Hefei, Anhui 230001, P.R. China

E-mail:1kma119@163.com

Key words: echocardiography, heart failure, ejection fraction, metoprolol, atrial fibrillation
AF incidence rate of $0.77 \%$ in the Chinese population (2). AF can lead to significant rates of morbidity [e.g., the development of apoplexia, embolism and heart failure (HF)] and mortality (3). Conversely, HF is also considered an etiological factor contributing to and exacerbating AF (4). Chronic HF is a group of syndromes which are characterised by cardiac dystrophy and declining heart function (5). Clinically, chronic $\mathrm{HF}$ is associated with ventricular filling disorder and a reduced cardiac ejection fraction (EF) (6). The European Society of Cardiology has characterised HF using left ventricular EF as follows: When the EF value is $\geq 50 \%$, patients are classified as having heart failure with preserved $\mathrm{EF}$, whereas when the EF value is $<50 \%$, patients are classified as having heart failure with reduced $\mathrm{EF}$ (6). Patients with chronic $\mathrm{HF}$ and $\mathrm{AF}$ have a greater risk of thromboembolic complications (e.g., cerebral stroke) (7). There are a number of pharmacological treatments available for HF and AF, such as $\beta$-blockers (8), angiotensin-converting enzyme inhibitors (9), angiotensin receptor blockers (10) and mineralocorticoid receptor antagonists (11). Clinically, $\beta$-blockers are the most preferred for the improvement of the prognosis, the prevention of arrhythmias, the improvement of left ventricular EF, the control of heart rate and the reduction of mortality of patients. Previous large-scale clinical studies conducted on patients with or without AF have confirmed the effectiveness of $\beta$-blockers for improving $\mathrm{HF}$ in patients with preserved EF $(11,12)$. However, the role of metoprolol in patients with a reduced EF and persistent AF has yet to be determined $(13,14)$. Therefore, the aim of this study was to retrospectively determine the effects of the sustained administration of metoprolol on patients with chronic AF and $\mathrm{HF}$ either with or without reduced EF.

\section{Patients and methods}

Patient information and follow-up. The Ethics Committee of the First Affiliated Hospital of the University of Science and Technology of China approved this retrospective medical record review (approval no. NCT02309398). From the history reports of the patients who were enrolled in this study, all patients or patient carers signed informed consents. Analyses were based on data from the Improving Care for Cardiovascular Disease in China (CCC) project, which is a collaborative project of the American Heart Association (AHA) and the Chinese Society of Cardiology (CSC). Data 
were collected prospectively for an investigator-initiated single centre study at The First Affiliated Hospital of the University of Science and Technology of China (UTSC). Chinese patients (aged >40 years) with persistent AF between 2015 and 2016 were treated with a single $47.5 \mathrm{mg}$ dose of metoprolol once per day for 2 years were selected for study. The inclusion criteria were as follows: i) Patients classified as Class II, III, or IV according to the New York Heart Association Functional Classification system (15); ii) diagnosed with persistent AF and HF presenting with either a reduced EF $(<50 \%)$ or preserved EF ( $\geq 50 \%)$. The exclusion criteria were as follows: i) incomplete $\beta$-blocker administration data; ii) had contraindication for $\beta$-blockers; iii) had their $\beta$-blockers discontinued. The total number of enrolled patients was 151, of which 47 presented with a reduced EF and 104 presented with a preserved EF. However, 15 patients were lost due to mortality during the follow-up period (5 in the reduced EF group and 10 in the preserved EF group). During the follow-up period, patient heart rates were brought to $<110$ beats per min according to the European Society of Cardiology Guidelines (7), using doses of metoprolol specifically determined for each individual patient by outpatient doctors or through telephone counselling. The following indicators were examined using echocardiography: Left ventricular EF, left ventricular end diastolic diameter and left atrial diameter. N-terminal pro b-type natriuretic peptide (NT-pro BNP) and 6-min walk tests (6MWT) were also performed during follow-up. 6MWTs were carried out according to the American Thoracic Society (ATS) guidelines (16). Briefly, patients were instructed to walk as far as possible up and down a 30-meter corridor for 6 min under the supervision of a physiotherapist. The physiotherapist encouraged the subject with standardised statements, such as 'you are doing well' and 'keep up the good work' and was asked to refrain from using other phrases. The patient or the physiotherapist could interrupt the 6MWT if the following symptoms appeared: Chest pain, intolerable dyspnoea, leg cramps, staggering, diaphoresis, or a pale or ashen appearance. All 6MWTs were administered by the same individual. The measurements included the following: A 6-min walking distance. Echocardiography and blood pressure measurements were performed before and after treatment using standard techniques by trained and experienced personnel.

Statistical analysis. Statistical analyses were performed using IBM SPSS Statistics 23 software (IBM Corp.). The approximate normal distribution of data was assessed by visual (histograms and normal Q-Q plots) and numerical investigative means (z-value of skewness and kurtosis; P-value of Shapiro-Wilk test). The analyses of patient characteristics and cardiac parameters between the cohorts was performed using Chi-square tests and independent sample t-tests. The analyses of cardiac parameters at baseline and after 2 years of metoprolol treatment were performed using paired sample t-tests. All tests were two-sided, with P-values $<0.05$ considered to indicate statistically significant differences.

\section{Results}

In total, 136 patients completed this 2-year study (151 original enrolments with 15 lost to mortality during the follow-up period). Of these, 42 had EF values $<50 \%$ and 94 presented with preserved EF values ( $\geq 50 \%$ ). No significant demographic or physical characteristic differences were observed between these 2 groups (Table I).

As measured by echocardiography, atrial and ventricular enlargement are characteristics of $\mathrm{AF}$ and $\mathrm{HF}$, respectively (Table II). In the low EF group, the patient baseline cardiac parameters prior to metoprolol treatment exhibited a statistically significant elevation in diastolic blood pressure, as well as statistically significant elevations in left ventricular and left atrial diameter compared to the preserved EF group. Additionally, the NT-pro BNP values were greater in the patients in the low EF group compared to the preserved EF group, and the $6 \mathrm{MWT}$ results were diminished in the low EF group compared to the patients with preserved EF function. The difference between the EF values between the 2 study groups was also statistically significant.

Following 2 years of treatment with metoprolol, patients with reduced EF values $<50 \%$ (Table III) exhibited significantly reduced systolic and diastolic blood pressures and heart rate compared to the baseline levels. These findings are typical of the effects observed following the administration of $\beta$-blockers. Notably, left ventricular diameter decreased, the EF value increased, and 6MWT results improved following treatment.

As regard patients presenting with preserved EF ( $\geq 50 \%)$ (Table IV) during baseline examinations, treatment with metoprolol significantly reduced the systolic and diastolic blood pressures and heart rate, as expected following $\beta$-blocker administration. However, the left ventricular and atrial diameters increased, the NT-pro BNP values increased, EF decreased, and the 6MWT results decreased.

Cardiac parameters of patients at the 2-year follow-up following metoprolol treatment, with comparisons between the two cohorts ( $\mathrm{EF}<50 \%$ and $\mathrm{EF} \geq 50 \%$ ) being performed (Table V). At the 2-year follow-up the patients with preserved EF exhibited cardia parameters which were statistically significantly superior to those of the the reduced EF group for all ultrasonic-derived cardiac parameters (e.g., left ventricle and atrial diameters, EF) NT-proBNP and the 6-min walk test.

\section{Discussion}

The aim of this study was to determine the role of metoprolol in adult Chinese patients with persistent AF, but with either reduced or preserved EF. $\beta$-blockers are widely used for the treatment and management of cardiac disorders (e.g., arrhythmia, reduced left ventricular EF, cardiac rate abnormalities, hypertension and HF) (17). $\beta$-blockers are preferred due to their inhibitory effects on the sympathetic nervous system, which translates into a beneficial effect for cardiac disorders, including HF (18-21). However, the long term effects of $\beta$-blockers on HF remain controversial (22-24).

The SENIORS trial noted that adult patients with HF and differing degrees of EF treated with nebivolol (a $\beta 1$ receptor blocker with nitric oxide-potentiating vasodilatory effects) failed to report any significant effect onmortality and cardiovascular hospital admission (25). In a pilot study, Mittal et al performed an investigator-initiated, randomised, double-blind, placebo-controlled, 14-week pilot study with 
Table I. Demographic and physical characteristics of the study subjects.

\begin{tabular}{lccc}
\hline Characteristics & EF <50\% (n=42) & EF $\geq 50 \%(\mathrm{n}=94)$ & P-value \\
\hline Sex, male, $\mathrm{n}(\%)$ & $24(57.1)$ & $47(50.0)$ & 0.441 \\
Age (years) & $72.9 \pm 9.9$ & $71.1 \pm 8.3$ & 0.297 \\
Weight (kg) & $66.2 \pm 13.3$ & $64.9 \pm 10.5$ & 0.531 \\
Height (cm) & $165.0 \pm 8.0$ & $165.1 \pm 8.3$ & 0.915 \\
Hypertension, n (\%) & $29(69.0)$ & $55(58.5)$ & 0.243 \\
Diabetes, n (\%) & $7(16.7)$ & $15(16.0)$ & 0.917 \\
Ischemic cardiomyopathy, n (\%) & $1(4.2)$ & $2(2.1)$ & 0.926 \\
Coronary heart disease, $\mathrm{n}(\%)$ & $11(26.2)$ & $15(16.0)$ & 0.161 \\
Congestive heart failure, $\mathrm{n}(\%)$ & $14(23.0)$ & $17(18.7)$ & 0.050
\end{tabular}

Comparisons between the 2 cohorts ( $\mathrm{EF}<50 \%$ and $\mathrm{EF} \geq 50 \%$ ) were made using Chi-square tests and independent samples t-tests. All discrete data are expressed as $\mathrm{n}(\%$ of total) and all continuous data are expressed as the means $\pm \mathrm{SD}$. EF, ejection fraction.

Table II. Patient baseline cardiac parameters before metoprolol treatment.

\begin{tabular}{lccr}
\hline Baseline parameters (pre-treatment) & EF $<50 \%(\mathrm{n}=42)$ & EF $\geq 50 \%(\mathrm{n}=94)$ & P-value \\
\hline Systolic pressure (mmHg) & $136.2 \pm 23.5$ & $135.5 \pm 20.2$ & 0.860 \\
Diastolic pressure (mmHg) & $85.6 \pm 15.5$ & $79.4 \pm 13.8$ & 0.022 \\
Heart rate (beats/min) & $90.3 \pm 23.0$ & $84.3 \pm 18.7$ & 0.112 \\
Left ventricle diameter (mm) & $66.4 \pm 7.6$ & $55.0 \pm 6.3$ & $<0.001$ \\
Left atrium diameter (mm) & $51.5 \pm 6.1$ & $47.3 \pm 6.4$ & 0.001 \\
Ejection fraction (\%) & $39.6 \pm 7.1$ & $62.7 \pm 7.5$ & $<0.001$ \\
NT-proBNP (pg/ml) & $3,454.7 \pm 1,254.6$ & $633.5 \pm 519.7$ & $<0.001$ \\
6-min walk test distance (m) & $201.9 \pm 86.7$ & $322.3 \pm 112.6$ & $<0.001$ \\
\hline
\end{tabular}

Comparisons between the 2 groups ( $\mathrm{EF}<50 \%$ and $\mathrm{EF} \geq 50 \%$ ) were performed using independent-samples t-tests. All data are expressed as the means $\pm \mathrm{SD}$. EF, ejection fraction; NT-proBNP, N-terminal pro b-type natriuretic peptide.

Table III. Cardiac parameters before and after metoprolol treatment for patients with a reduced EF.

\begin{tabular}{lccc}
\hline Parameters for EF $<50 \%(\mathrm{n}=42)$ & At baseline & At 2-year follow-up & P-value \\
\hline Systolic pressure $(\mathrm{mmHg})$ & $136.2 \pm 23.5$ & $127.6 \pm 13.4$ & $<0.001$ \\
Diastolic pressure $(\mathrm{mmHg})$ & $85.6 \pm 15.5$ & $76.6 \pm 10.0$ & $<0.001$ \\
Heart rate (beats/min) & $90.3 \pm 23.0$ & $65.0 \pm 7.5$ & $<0.001$ \\
Left ventricular diameter $(\mathrm{mm})$ & $66.4 \pm 7.6$ & $52.0 \pm 5.2$ & 0.001 \\
Left atrial diameter (mm) & $51.5 \pm 6.1$ & $43.7 \pm 7.8$ & 0.264 \\
EF (\%) & $39.6 \pm 7.1$ & $3,350 \pm 2,452.7$ & $<0.001$ \\
NT-proBNP (pg/ml) & $3,454.7 \pm 1,254.6$ & $238.5 \pm 89.7$ & 0.790 \\
6-min walk test distance (m) & $201.9 \pm 86.7$ & 0.008 \\
\hline
\end{tabular}

Comparisons between the time points was performed using paired samples t-tests. All data are expressed as the means \pm SD. EF, ejection fraction; NT-proBNP, N-terminal pro b-type natriuretic peptide.

metoprolol succinate as a study drug for patients with heart failure with preserved EF. The results of the pilot study revealed that metoprolol administration yielded some benefits for patients with HF with preserved EFs, as reflected by improvements in echocardiographic and biochemical parameters (26). A meta-analysis was published to clarify whether any $\beta$-blocker was superior in patients with HF and reduced EF. This analysis included 21 trials and found that 
Table IV. Cardiac parameters before and after metoprolol treatment for patients with preserved ejection fraction.

\begin{tabular}{lccr}
\hline Parameters for EF $\geq 50 \%(\mathrm{n}=94)$ & At baseline & At 2-year follow-up & P-value \\
\hline Systolic pressure $(\mathrm{mm} \mathrm{Hg})$ & $135.5 \pm 20.2$ & $127.1 \pm 12.7$ & $<0.001$ \\
Diastolic pressure $(\mathrm{mm} \mathrm{Hg})$ & $79.4 \pm 13.8$ & $74.0 \pm 9.2$ & $<0.001$ \\
Heart rate (beats/min) & $84.3 \pm 18.7$ & $75.8 \pm 9.9$ & $<0.001$ \\
Left ventricular diameter (mm) & $55.0 \pm 6.3$ & $57.1 \pm 5.4$ & $<0.001$ \\
Left atrial diameter (mm) & $47.3 \pm 6.4$ & $50.0 \pm 7.1$ & $<0.001$ \\
EF (\%) & $62.7 \pm 7.5$ & $54.3 \pm 5.2$ & $<0.001$ \\
NT-proBNP (pg/ml) & $633.5 \pm 519.7$ & $1,998.7 \pm 1,242.7$ & $<0.001$ \\
6-min walk test distance $(\mathrm{m})$ & $322.3 \pm 112.6$ & $304.6 \pm 109.1$ & 0.038 \\
\hline
\end{tabular}

Comparisons between the time points was performed using paired samples t-tests. All data are expressed as the means \pm SD. EF, ejection fraction; NT-proBNP, N-terminal pro b-type natriuretic peptide.

Table V. Cardiac parameters of patients at 2-year follow-up following metoprolol treatment.

\begin{tabular}{lccr}
\hline Parameters at 2-year follow-up & EF $<50 \%(\mathrm{n}=42)$ & EF $\geq 50 \%(\mathrm{n}=94)$ & P-value \\
\hline Systolic pressure (mm Hg) & $127.6 \pm 13.4$ & $127.1 \pm 12.7$ & 0.823 \\
Diastolic pressure (mm Hg) & $76.6 \pm 10.0$ & $74.0 \pm 9.2$ & 0.144 \\
Heart rate (beats/min) & $77.8 \pm 10.5$ & $75.8 \pm 9.9$ & 0.293 \\
Left ventricle diameter, (mm) & $65.0 \pm 7.5$ & $57.1 \pm 5.4$ & $<0.001$ \\
Left atrial diameter, (mm) & $52.0 \pm 5.2$ & $50.0 \pm 7.1$ & 0.106 \\
Ejection fraction, (\%) & $43.7 \pm 7.8$ & $54.3 \pm 5.2$ & $<0.001$ \\
NT-proBNP (pg/ml) & $3,350 \pm 2,452.7$ & $1,998.7 \pm 1,242.7$ & $<0.001$ \\
6-min walk test distance (m) & $238.5 \pm 89.7$ & $304.6 \pm 109.1$ & 0.001 \\
\hline
\end{tabular}

Comparisons were made between the two cohorts ( $\mathrm{EF}<50 \%$ and $\mathrm{EF} \geq 50 \%)$ using independent samples $\mathrm{t}$-tests. All data are expressed as the means $\pm \mathrm{SD}$.

the benefit of $\beta$-blockers in patients with $\mathrm{HF}$ and a reduced EF was primarily due to a class effect, as no statistical evidence supported the superiority of any single drug over the others (22). Published research has also focused on the effects of $\beta$-blockers on patients with HF with preserved $\mathrm{EF}$ and AF. Indeed, it has been shown that metoprolol is beneficial for all patients, even in patients with a reduced EF. Selective $\beta$-blockers inhibit the sinus node, ultimately leading to the control of the atrioventricular node, which is responsible for AF. Hence, the selective inhibition of $\beta 1$ receptors helps preserve cardiac function in $\mathrm{HF}$ (21).

BNP or NT-pro BNP are biomarkers detected by tests used to aid in the detection, diagnosis and the evaluation of the severity of HF. The NT-pro BNP levels were measured in this study; due to high variability in the reduced EF group, no statistically significant difference was found between the baseline and post-treatment time points. In the preserved EF group, however, a statistically significant increase in NT-pro BNP was observed after 2 years of treatment, which was consistent with the worsening HF in this patient cohort, as indicated by other cardiac parameters (e.g., enlarged left ventricle/atria and reduced $\mathrm{EF}$ ). When comparing the patients with preserved and reduced EF at the 2 year follow-up, the patients with preserved EF exhibited values which were statistically significant superior for all ultrasonic derived cardiac parameters, NT-pro BNP and the 6MWT than the reduced EF group.

Previous studies including meta-analyses on the use of $\beta$-blockers have not reported a reduced mortality rate of patients with HF with preserved EF $(12,25,27)$. The present study highlights the importance of efficient metoprolol treatment in adult patients with chronic AF and reduced EF, as well as the beneficial effects on cardiac parameters resulting from such a treatment. Left ventricular enlargement leads to increased end diastolic and systolic volumes and a reduced cardiac output. A reduction in diastolic blood pressure favours protection against left ventricular enlargement, while a decrease in systolic pressure may increase the pumping efficiency of the heart. This study confirmed that metoprolol treatment resulted in a desired negative chronotropic effect that is essential for reducing the workload of the failing heart. Metoprolol treatment significantly reduced left atrial and left ventricular diameters, indicating that metoprolol reverses some of the pathophysiological changes observed in patients with AF and HF. However, metoprolol treatment in patients with preserved EF did not present the same effects; indeed, several important cardiac parameters worsened, including EF, which diminished. It is not clear whether these findings are 
due to the cardiac function natural histories in these patients presenting with $\mathrm{AF}$ and preserved $\mathrm{EF}$, or whether the drug itself had a deleterious or non-protective effect, as this study lacked an untreated control group for a statistical comparison. Beyond this, this study was also hampered by the small sample size. Nonetheless, the sample size was still sufficient for the identification of statistically significant associations using paired-sample t-tests.

In conclusion, this study demonstrated a beneficial effect for long-term sustained-release metoprolol treatment in adult Chinese patients with persistent AF and reduced EF. Large-scale clinical trials with patients with HF presenting with AF and varying degrees of EF are still required to determine whether the mortality rate is also reduced.

\section{Acknowledgements}

Not applicable.

\section{Funding}

The CCC-ACS project is a collaborative study of the American Heart Association (AHA) and Chinese Society of Cardiology; the AHA has been funded by Pfizer for quality improvement initiatives through an independent grant for learning and change. This study was also supported by the Anhui Province Science and Technology Program (no. 1604a0802074).

\section{Availability of data and materials}

All data generated or analysed during this study are included in this published article or are available from the corresponding author on reasonable request.

\section{Authors' contributions}

WC and LM conceived and designed the study, provided study materials or patients and were responsible for the collection and assembly of the data, data analysis and interpretation. WC was involved in the writing of the manuscript. LM was involved in the editing of the manuscript. All authors have read and approved the final manuscript.

\section{Ethics approval and consent to participate}

The Ethics Committee of the First Affiliated Hospital of the University of Science and Technology of China approved this retrospective medical record review (approval no. NCT02309398). From the history reports of the patients who were enrolled in this study, all patients or patient carers signed informed consents. Please kindly check this situation with the Regional Ethics Committee of our hospital if it is necessary.

\section{Patient consent for publication}

Not applicable.

\section{Competing interests}

The authors declare that they have no competing interests.

\section{References}

1. Kannel WB, Wolf PA, Benjamin EJ and Levy D: Prevalence, incidence, prognosis, and predisposing conditions for atrial fibrillation: Population-based estimates. Am J Cardiol 82 (8A): 2N-9N, 1998.

2. Feinberg WM, Blackshear JL, Laupacis A, Kronmal R and Hart RG: Prevalence, age distribution, and gender of patients with atrial fibrillation. Analysis and implications. Arch Intern Med 155: 469-473, 1995.

3. Benjamin EJ, Wolf PA, D'Agostino RB, Silbershatz H, Kannel WB and Levy D: Impact of atrial fibrillation on the risk of death: The Framingham Heart Study. Circulation 98: 946-952, 1998.

4. Anter E, Jessup M and Callans DJ: Atrial fibrillation and heart failure: Treatment considerations for a dual epidemic. Circulation 119: 2516-2525, 2009.

5. Hoppe UC, Casares JM, Eiskjaer H, Hagemann A, Cleland JGF, Freemantle $\mathrm{N}$ and Erdmann E: Effect of cardiac resynchronization on the incidence of atrial fibrillation in patients with severe heart failure. Circulation 114: 18-25, 2006.

6. Gazewood JD and Turner PL: Heart Failure with Preserved Ejection Fraction: Diagnosis and Management. Am Fam Physician 96: 582-588, 2017.

7. Kirchhof P, Benussi S, Kotecha D, Ahlsson A, Atar D, Casadei B, Castellá M, Diener HC, Heidbuchel H, Hendriks J, et al: 2016 ESC Guidelines for the Management of Atrial Fibrillation Developed in Collaboration With EACTS. Rev Esp Cardiol (Engl Ed) 70: 50, 2017.

8. Swedberg K, Olsson LG, Charlesworth A, Cleland J, Hanrath P, Komajda M, Metra M, Torp-Pedersen C and Poole-Wilson P: Prognostic relevance of atrial fibrillation in patients with chronic heart failure on long-term treatment with beta-blockers: Results from COMET. Eur Heart J 26: 1303-1308, 2005.

9. Pedersen OD, Bagger H, Kober L and Torp-Pedersen C: Trandolapril reduces the incidence of atrial fibrillation after acute myocardial infarction in patients with left ventricular dysfunction. Circulation 100: 376-380, 1999.

10. Ducharme A, Swedberg K, Pfeffer MA, Cohen-Solal A, Granger CB, Maggioni AP, Michelson EL, McMurray JJ, Olsson L, Rouleau JL, et al; CHARM Investigators: Prevention of atrial fibrillation in patients with symptomatic chronic heart failure by candesartan in the Candesartan in Heart failure: Assessment of Reduction in Mortality and morbidity (CHARM) program. Am Heart J 152: 86-92, 2006.

11. McMurray J, Køber L, Robertson M, Dargie H, Colucci W, Lopez-Sendon J, Remme W, Sharpe DN and Ford I: Antiarrhythmic effect of carvedilol after acute myocardial infarction: Results of the Carvedilol Post-Infarct Survival Control in Left Ventricular Dysfunction (CAPRICORN) trial. J Am Coll Cardiol 45: 525-530, 2005.

12. Kotecha D, Holmes J, Krum H, Altman DG, Manzano L, Cleland JG, Lip GY, Coats AJ, Andersson B, Kirchhof P, et al; Beta-Blockers in Heart Failure Collaborative Group: Efficacy of $\beta$ blockers in patients with heart failure plus atrial fibrillation: An individual-patient data meta-analysis. Lancet 384: 2235-2243, 2014.

13. Piccini JP, Connolly SJ, Abraham WT, Healey JS, Steinberg BA, Al-Khalidi HR, Dignacco P, van Veldhuisen DJ, Sauer WH, White M, et al: A genotype-directed comparative effectiveness trial of Bucindolol and metoprolol succinate for prevention of symptomatic atrial fibrillation/atrial flutter in patients with heart failure: Rationale and design of the GENETIC-AF trial. Am Heart J 199: 51-58, 2018.

14. Silvet H, Hawkins LA and Jacobson AK: Heart rate control in patients with chronic atrial fibrillation and heart failure. Congest Heart Fail 19: 25-28, 2013.

15. Dolgin Martin (ed): Nomenclature and criteria for diagnosis of diseases of the heart and great vessels/the Criteria Committee of the New York Heart Association. 9th edition. Little, Brown, Boston, 1994.

16. ATS Committee on Proficiency Standards for Clinical Pulmonary Function Laboratories: ATS statement: Guidelines for the six-minute walk test. Am J Respir Crit Care Med 166: 111-117, 2002.

17. Bristow MR: Treatment of chronic heart failure with $\beta$-adrenergic receptor antagonists: A convergence of receptor pharmacology and clinical cardiology. Circ Res 109: 1176-1194, 2011. 
18. McMurray JJV, Adamopoulos S, Anker SD, Auricchio A, Böhm M, Dickstein K, Falk V, Filippatos G, Fonseca C, Gomez-Sanchez MA, et al: Task Force for the Diagnosis and Treatment of Acute and Chronic Heart Failure 2012 of the European Society of Cardiology; ESC Committee for Practice Guidelines: ESC guidelines for the diagnosis and treatment of acute and chronic heart failure 2012: The Task Force for the Diagnosis and Treatment of Acute and Chronic Heart Failure 2012 of the European Society of Cardiology. Developed in collaboration with the Heart Failure Association (HFA) of the ESC. Eur J Heart Fail 14: 803-869, 2012.

19. Hunt SA, Abraham WT, Chin MH, Feldman AM, Francis GS, Ganiats TG, Jessup M, Konstam MA, Mancini DM, Michl K, et al; American College of Cardiology Foundation; American Heart Association: 2009 Focused update incorporated into the ACC/AHA 2005 Guidelines for the Diagnosis and Management of Heart Failure in Adults A Report of the American College of Cardiology Foundation/American Heart Association Task Force on Practice Guidelines Developed in Collaboration With the International Society for Heart and Lung Transplantation. J Am Coll Cardiol 53: e1-e90, 2009.

20. Lindenfeld J, Albert NM, Boehmer JP, Collins SP, Ezekowitz JA, Givertz MM, Katz SD, Klapholz M, Moser DK, Rogers JG, et al Heart Failure Society of America: HFSA 2010 Comprehensive Heart Failure Practice Guideline. J Card Fail 16: e1-e194, 2010.

21. Miller RJH, Howlett JG, Chiu MH, Southern DA, Knudtson M and Wilton SB: Relationships among achieved heart rate, $\beta$-blocker dose and long-term outcomes in patients with heart failure with atrial fibrillation. Open Heart 3: e000520, 2016.

22. Chatterjee S, Biondi-Zoccai G, Abbate A, D'Ascenzo F, Castagno D, Van Tassell B, Mukherjee D and Lichstein E: Benefits of $\beta$ blockers in patients with heart failure and reduced ejection fraction: Network meta-analysis. BMJ 346 (jan16 1): f55, 2013.
23. Edelmann F, Musial-Bright L, Gelbrich G, Trippel T, Radenovic S, Wachter R, Inkrot S, Loncar G, Tahirovic E, Celic V, et al; CIBIS-ELD Investigators and Project Multicenter Trials in the Competence Network Heart Failure: Tolerability and Feasibility of Beta-Blocker Titration in HFpEF Versus HFrEF: Insights From the CIBIS-ELD Trial. JACC Heart Fail 4: 140-149, 2016.

24. Yamamoto $\mathrm{K}$, Origasa $\mathrm{H}$ and Hori $\mathrm{M}$; J-DHF Investigators: Effects of carvedilol on heart failure with preserved ejection fraction: The Japanese Diastolic Heart Failure Study (J-DHF). Eur J Heart Fail 15: 110-118, 2013.

25. Flather MD, Shibata MC, Coats AJS, Van Veldhuisen DJ, Parkhomenko A, Borbola J, Cohen-Solal A, Dumitrascu D, Ferrari R, Lechat P, et al; SENIORS Investigators: Randomized trial to determine the effect of nebivolol on mortality and cardiovascular hospital admission in elderly patients with heart failure (SENIORS). Eur Heart J 26: 215-225, 2005.

26. Mittal N, Shafiq N, Reddy S, Malhotra S, Kumari S and Varma S: Evaluation of efficacy of metoprolol in patients having heart failure with preserved ejection fraction: A randomized, double-blind, placebo-controlled pilot trial. Perspect Clin Res 8: 124-131, 2017.

27. Hernandez AF, Hammill BG, O'Connor CM, Schulman KA, Curtis LH and Fonarow GC: Clinical effectiveness of beta-blockers in heart failure: Findings from the OPTIMIZE-HF (Organized Program to Initiate Lifesaving Treatment in Hospitalized Patients with Heart Failure) Registry. J Am Coll Cardiol 53: 184-192, 2009.

This work is licensed under a Creative Commons Attribution-NonCommercial-NoDerivatives 4.0 International (CC BY-NC-ND 4.0) License. 